Rev. Inst. Flor. v. 26 n. 2 p. 183-191 dez. 2014

http://dx.doi.org/10.4322/rif.2014.014

ISSN impresso 0103-2674/on-line 2178-5031

\title{
ALTERAÇÕES DA RELAÇÃO C/N DE UM LATOSSOLO VERMELHO-AMARELO SOB DIFERENTES COBERTURAS VEGETAIS EM BRASNORTE - MT ${ }^{1}$ \\ CHANGES IN C/N RELATION OF A RED-YELLOW LATOSOL UNDER DIFFERENT PLANT CULTIVATIONS IN BRASNORTE - MT
}

\author{
Cristiane Ramos VIEIRA ${ }^{2,5}$; Oscarlina Lúcia dos Santos WEBER $^{3}$; \\ Rafael Ferreira LIUTI²; José Fernando SCARAMUZZA ${ }^{4}$
}

\begin{abstract}
RESUMO - Este estudo objetivou analisar as alterações nos teores de carbono e de nitrogênio e na relação $\mathrm{C} / \mathrm{N}$ em solos sob diferentes coberturas. Os sistemas avaliados foram: Floresta Secundária, pastagem, e plantios de teca (Tectona grandis) com 8, 13, 16 e 19 anos, utilizando o delineamento inteiramente casualizado para a coleta do solo nas profundidades 0 a 5,5 a 10 e 10 a $20 \mathrm{~cm}$. Nas áreas dos plantios de $T$. grandis as amostras de solo foram coletadas na projeção da copa e nas entrelinhas, e nas áreas de pastagem e de Floresta Secundária a coleta foi aleatória. Verificou-se que os teores de carbono orgânico total - COT foram mais elevados nas primeiras camadas de solo, diminuindo conforme a profundidade. A manutenção dos teores de $\mathrm{C}$ e de N, semelhantes aos da Floresta Secundária, permitem caracterizar o solo dos sistemas pastagem e de cultivo de T. grandis como quimicamente não degradados, sendo que os valores de relação $\mathrm{C} / \mathrm{N}$ indicam que o processo dominante em todos os sistemas foi a mineralização.
\end{abstract}

Palavras-chave: sistemas de manejo; estoque de carbono; estoque de nitrogênio; matéria orgânica.

\begin{abstract}
This study aimed to analyze the changes in the levels of carbon and nitrogen and $\mathrm{C} / \mathrm{N}$ relation in different soil covers. The systems evaluated were: Secondary Forest, pasture, and plantations of teak at 8,13,16 and 19 years old, using a completely randomized design in split plot to collect soil in three depths $(0-5,5-10$ and 10-20 cm). In the area of teak plantations samples of soil were collected on the projection of the tree crown and between lines, in the pasture and Secondary Forest they were collected randomly. It was found that the concentration of total organic carbon - TOC was higher in the first depth, decreasing as the depth. The maintenance of the levels of $\mathrm{C}$ and $\mathrm{N}$ similar to Secondary Forest can characterize the soil of grass pastures systems and cultivation of teak as chemical not degraded and $\mathrm{C} / \mathrm{N}$ relation indicates that the dominant process on all systems was the mineralization.
\end{abstract}

Keywords: management systems; carbon stock; nitrogen stock; organic matter.

\footnotetext{
${ }^{1}$ Recebido para análise em 11.08.14. Aceito para publicação em 06.11.14.

${ }^{2}$ Universidade Federal de Mato Grosso, Av. Fernando Corrêa da Costa, n. 2367, Bairro Boa Esperança, 78060-900 Cuiabá, MT, Brasil.

${ }^{3}$ Universidade Federal de Mato Grosso, Departamento de Solos e Engenharia Rural, Av. Fernando Corrêa da Costa, n. 2367, Bairro Boa Esperança, 78060-900 Cuiabá, MT, Brasil. oscsan@uol.com.br.

${ }^{4}$ Universidade Federal de Mato Grosso, Departamento de Solos e Engenharia Rural, Av. Fernando Corrêa da Costa, n. 2367, Bairro Boa Esperança, 78060-900 Cuiabá, MT, Brasil. jscaramuzza@uol.com.br.

${ }^{5}$ Autor para correspondência: Cristiane Ramos Vieira - cris00986@hotmail.com
} 


\section{INTRODUÇÃO}

O Bioma Cerrado passa por intensa transformação da paisagem para dar suporte às grandes áreas de monocultivos. Nesse contexto, situa-se Brasnorte, município localizado na região norte de Mato Grosso e cujas principais atividades econômicas se baseiam na produção agrícola, pecuária e exploração madeireira em florestas nativa e plantada.

Em condições nativas, o solo organiza-se em uma estrutura bem definida pela sua composição granulométrica, química e atuação dos agentes biológicos. Sua conversão para agricultura convencional impõe mudanças drásticas nessa estabilidade, refletindo-se na perda da matéria orgânica - MO e dos agregados mais complexos (Bayer et al., 2006). De acordo com Matias et al. (2012), o intenso revolvimento do sistema de plantio convencional favorece a decomposição de $\mathrm{MO}$, com efeitos negativos sob os atributos físicos no solo, pois a redução da matéria orgânica do solo - MOS ocasiona redução nos teores de C e de N, também observado por Souza et al. (2006); Matias et al. (2012) e Loss et al. (2013).

Segundo Denardin et al. (2014), o manejo inadequado do solo, ou a alteração da cobertura florestal, levam a grandes perdas do $\mathrm{C}$ estocado, em pouco tempo. Dessa maneira, o solo, considerado um reservatório ou um dreno de $\mathrm{C}$, pode tornar-se fonte de C para a atmosfera, aumentando a concentração dos gases do efeito estufa. Enquanto as perdas de $\mathrm{N}$ acarretam em reduções no metabolismo das plantas e, consequentemente, no crescimento, comprometendo a produtividade de todo o cultivo. Portanto, o estoque de $\mathrm{C}$ de um solo sob vegetação natural representa o balanço entre a adição de material vegetal morto e a perda pela decomposição ou mineralização (Machado, 2005).

Segundo Santos (2008), uma alternativa para manter ou melhorar os estoques de MOS, de $\mathrm{C}$, de $\mathrm{N}$ e a fertilidade é a utilização de sistemas de culturas que adicionam material orgânico. A manutenção dos resíduos culturais na superfície proporciona decomposição gradual e acúmulo do material orgânico no perfil do solo (Franzluebbers et al., 2007). Campos et al. (2013) observaram que os sistemas de plantio direto acumularam maiores teores de carbono orgânico total - COT e nitrogênio total - NT no perfil do solo, após cinco anos de implantação, em razão da maior estabilidade das frações húmicas e menor grau de mineralização da MOS.

No entanto, resultados contraditórios têm sido observados, demonstrando que estes dependerão da cultura implantada, do solo e do bioma utilizado como referência. Pulrolnik et al. (2009) observaram que o cultivo do Eucalyptus não reduziu o estoque de $\mathrm{C}$ e de $\mathrm{N}$ da biomassa microbiana do solo em comparação com a do Cerrado e pastagem, contribuindo para o aumento da MOS. Moreira e Malavolta (2004) concluíram que a sucessão floresta primária-pastagem-cupuaçuzal causa diminuição significativa da $\mathrm{MO}$ e do $\mathrm{C}$ da biomassa microbiana do solo. No entanto, a floresta primária apresentou maior relação $\mathrm{C} / \mathrm{N}$ da biomassa, o que resultou em menor perda de $\mathrm{C}$.

Enquanto Campolin (2006) observou que a conversão do Cerrado em plantios florestais de $T$. grandis alterou a capacidade de armazenamento de MOS, mantendo ou elevando os teores e estoques de MO no solo. Resultados contraditórios foram observados por Souza et al. (2006) ao verificarem que o estoque de $\mathrm{C}$ do solo reduziu em até $25 \%$ na área sob pastagem, em relação ao Cerrado, e a menor perda ocorreu na área sob sorgo em plantio direto $(4 \%)$.

Portanto, uma das preocupações atuais é o desenvolvimento de pesquisas que verifiquem a influência dos sistemas de cultivo na qualidade do solo, utilizando $\mathrm{C}$ e $\mathrm{N}$ como indicativos. Desse modo, o estudo visou quantificar os teores de $\mathrm{C}$ e de $\mathrm{N}$, e a relação $\mathrm{C} / \mathrm{N}$ em diferentes camadas do perfil do solo, sob diferentes sistemas de cultivo, utilizando a Floresta Secundária como referência.

\section{MATERIAL E MÉTODOS}

O experimento foi desenvolvido na Fazenda Paraná, propriedade da BERTeca Brasil, unidade industrial pertencente ao grupo Berneck S. A. Painéis e Serrados, localizada no município de Brasnorte, região centro-norte do Estado de Mato Grosso.

A propriedade onde foi realizado este trabalho está classificada e situada por Brasil (1982) como pertencente ao Grupo Parecis. A Chapada dos Parecis constitui-se, litologicamente, de arenitos do Grupo Parecis, 
VIEIRA, C.R. et al. Alterações $\mathrm{C} / \mathrm{N}$ de um Latossolo Vermelho-Amarelo sob diferenes coberturas vegetais

os quais apresentam acamamento plano-paralelo, caracterizando a homogeneidade topográfica desta subunidade. Possui uma camada de sedimentos finos recobrindo parcialmente o grupo. Essa camada compunha-se de um material concrecionário, sotoposto a um solo Latossolo Argilo-arenoso, em topografia suave, com relevo plano e suave ondulado.

O solo característico na região é do tipo Latossolo Vermelho-Amarelo - LVA, que são solos com teores de $\mathrm{Fe}_{2} \mathrm{O}_{3}$ iguais ou inferiores a $11 \%$ e, normalmente, acima de $7 \%$ quando argilosos ou muito argilosos e não concrecionários. São profundos ou muito profundos, bem drenados, com textura argilosa, muito argilosa ou média (Empresa Brasileira de Pesquisa Agropecuária EMBRAPA, 1999).

Para o estudo, foram escolhidas seis áreas contíguas: (I) cobertura vegetal de Floresta Secundária (FS); (II) pastagem extensiva com braquiária (Brachiaria brizantha (Hochst.) Stapf.) 29 anos (PA); (III) plantio de teca (Tectona grandis) com 19 anos (teca 19); (IV) plantio de teca com 16 anos (teca 16); (V) plantio de teca com 13 anos (teca 13) e teca com 8 anos (teca 8), considerando como tratamento cada um dos sistemas.

O Sistema FS está sob Floresta Ombrófila Aberta, cuja última interferência antrópica ocorreu no ano de 1999, com a retirada de espécies de alto valor comercial como: mogno (Swietenia macrophylla King), cerejeira (Amburana cearenses Fr. All), cedro (Cedrela fissilis Vell.) e peroba (Aspidosperma polyneuron Muell. Arg).

Para a implantação do sistema PA, a floresta primária foi inicialmente explorada de modo comercial. Logo após, implantou-se o método do "correntão" para a retirada total da vegetação restante, que foi leirada e queimada. A taxa de lotação é inferior a duas unidades animal por hectare, sendo a área utilizada na forma de rotativo não controlado. A área não recebe adubação, porém mantém a serapilheira.

A supressão da vegetação nas áreas onde posteriormente foram implantados os sistemas teca 19 , teca 13 e teca 8 foi realizada idem ao da pastagem. A diferença na implantação do sistema teca 16 está no cultivo de arroz em intervalo de três anos consecutivos anteriormente ao plantio, para cuja implantação se realizou a calagem e adubação NPK, porém sem controle quanto às características químicas do solo no momento, bem como as exigências da espécie.

Os sistemas teca 19 , teca 16 , teca 13 e teca 8 foram plantados com mudas provenientes de origem seminal (com variabilidade genética), produzidas em viveiro próprio do tipo toco raiz nua, formando área plantada de 764,33 hectares.

As coletas de solo ocorreram no mês de maio de 2011, no final da estação chuvosa, nas camadas de 0 a 5,5 a 10 e 10 a $20 \mathrm{~cm}$ de profundidade, a partir da abertura de três minitrincheiras de $0,5 \times 0,5 \mathrm{~m}$ e $30 \mathrm{~cm}$ de profundidade, para cada tratamento.

As coletas foram realizadas em locais distintos dentro dos sistemas FS e PA, demarcando uma área de 0,5 ha para cada sistema, com a abertura das minitrincheiras, aleatoriamente, com distância mínima de $5 \mathrm{~m}$ entre elas.

Para os sistemas teca 19 , teca 16 , teca 13 e teca 8 , as coletas foram realizadas em área total de 0,5 ha de plantio, com coletas na projeção da copa e nas entrelinhas, percorrendo a área em zigue-zague, com distância mínima de $5 \mathrm{~m}$ entre as minitrincheiras.

O delineamento utilizado foi o inteiramente casualizado, contendo três repetições em cada sistema de uso e tipo de amostra, cujos tratamentos consistiram em uma referência (T1 - Floresta Secundária) e cinco diferentes sistemas de uso: T2 - Pastagem - PA; T3 - Teca 19 anos coleta entrelinhas; T4 - Teca 16 anos coleta entrelinhas; T5 - Teca 13 anos coleta entrelinhas; T6 - Teca 8 anos coleta entrelinhas; T7 - Teca 19 anos coleta projeção de copa; T8 - Teca 16 anos coleta projeção de copa; T9 - Teca 13 anos coleta projeção de copa; T10 - Teca 8 anos coleta projeção de copa.

As amostras de solo foram secas ao ar e peneiradas em malha de $2 \mathrm{~mm}$. O carbono orgânico - CO do solo foi determinado pelo método descrito em Yeomans e Bremner (1988), enquanto o N, pelo método de Bremner e Mulvaney (1982). Os tratos silviculturais dentro das áreas de plantio de teca estão apresentados na Tabela 1.

Os dados foram submetidos ao teste Scott-Knott a 5\% de significância quando o teste $\mathrm{F}$ da ANOVA foi significativo, utilizando o software SISVAR 4.6 desenvolvido pela Universidade Federal de Lavras - UFLA (Ferreira, 2003). 
Tabela 1. Tratos silviculturais nos plantios de T. grandis.

Table 1. Silviculture management in T. grandis plantations.

\begin{tabular}{c|l}
\hline Sistema & \multicolumn{1}{c}{ Tratamentos Silviculturais } \\
\hline T19 & $\begin{array}{l}\text { Desbaste no ano de 1996, retirando 30\%, obedecendo a um desbaste baixo do tipo regular. } \\
\text { Desbaste no ano de 2002, retirando } 40 \% \text {, obedecendo a um desbaste sistemático (retirada da sétima } \\
\text { linha) em conjunto com um desbaste baixo. } \\
\text { Desbaste no ano de 2008, retirando 55\%, obedecendo a um desbaste baixo. }\end{array}$ \\
\hline T16 & $\begin{array}{l}\text { Desbaste no ano de 1999, retirando 25\%, obedecendo a um desbaste baixo do tipo regular. } \\
\text { Desbaste no ano de 2003, retirando 30\%, obedecendo a um desbaste sistemático (retirada da sétima linha). }\end{array}$ \\
\hline T13 & $\begin{array}{l}\text { Desbaste no ano de 2002, retirando 30\%, obedecendo a um desbaste baixo do tipo regular. } \\
\text { Desbaste no ano de 2007, retirando 40\%, obedecendo a um desbaste sistemático (retirada da sétima linha). }\end{array}$ \\
\hline T8 & Desbaste no ano de 2009, retirando 50\%, obedecendo a um desbaste baixo do tipo pesado. \\
\hline
\end{tabular}

\section{RESULTADOS E DISCUSSÃO}

\subsection{Carbono Orgânico Total}

A conversão da vegetação nativa em diferentes usos e manejo do solo acarretou alterações nos atributos químicos estudados $\mathrm{e}$ apresentados nas Tabelas de 2 a 4 . Os teores de carbono orgânico total - COT, nos diferentes sistemas de uso, encontram-se na Tabela 2. Ressalta-se que as coletas de solo na Floresta Secundária ocorreram 12 anos após a última intervenção. Os resultados obtidos nos sistemas de cultivo com pastagem e com $T$. grandis foram comparados com o sistema natural considerando essas condições, o que pode ter inferido nos teores de C, reduzindo-os, quando comparados com outras pesquisas.

Nas condições atuais do solo, nota-se que na camada 0 a $5 \mathrm{~cm}$ os sistemas de manejo com cultivos de $T$. grandis e a pastagem foram consideradas semelhantes ao sistema floresta, no que se refere aos teores de COT. As maiores diferenças entre sistemas ocorreram nas camadas mais profundas.

Em geral, os teores de COT no sistema floresta e na pastagem reduziram com o aumento da profundidade, o mesmo foi observado por Santos (2007), Campos et al. (2013) e Denardin et al. (2014). O teor mais elevado de COT, na primeira camada de solo, deve-se provavelmente à deposição de material orgânico na superfície do solo, como a presença de biomassa diminui com a profundidade, diminuem os teores de COT. $\mathrm{O}$ mesmo não foi observado para os sistemas com cultivo de $T$. grandis.

Nas camadas de 5 a $10 \mathrm{~cm}$ e 10 a $20 \mathrm{~cm}$, os sistemas: pastagem, teca 16 anos entrelinhas e teca 8 anos apresentaram teores de COT mais elevados que no sistema floresta. Segundo Castro (2008), isso pode ser explicado pela intensidade dos processos de adição de resíduos vegetais com baixas taxas de decomposição na superfície do solo, aumentando seu teor com a profundidade, porque a serapilheira formada pelo sistema teca é mais lignificada, demorando mais para decompor. Diferentemente do sistema de floresta, que possui diversidade de material a ser decomposto.

Ressalta-se que a Floresta Secundária apresentou redução significativa dos teores de COT conforme aumentou a profundidade; isto se deve à diversidade de material vegetal e, assim, de microrganismos, que possibilitam a degradação com maior rapidez que nos demais sistemas. Segundo Denardin et al. (2014), esses resultados demonstram a importância da estabilidade do solo nos sistemas florestais e do aporte de resíduos vegetais, pois o manejo do solo e a ação de fatores abióticos influenciam na degradação da $\mathrm{MO}$ e, assim, nos teores de $\mathrm{C}$, ficando evidente que os maiores teores e maiores perdas de $\mathrm{C}$ ocorrem nas camadas superficiais devido a maior presença de microrganismos. 
VIEIRA, C.R. et al. Alterações C/N de um Latossolo Vermelho-Amarelo sob diferenes coberturas vegetais

Tabela 2. Carbono orgânico total ( $\left(\mathrm{g}^{\mathrm{kg}}{ }^{-1}\right)$ em função dos sistemas de cultivo e das profundidades de amostragem.

Table 2. Total organic carbon ( $\left.\mathrm{g} \cdot \mathrm{kg}^{-1}\right)$ in function of cultivation systems and sampling depths.

\begin{tabular}{|c|c|c|c|c|}
\hline \multirow{2}{*}{ Tratamento } & $0-5 \mathrm{~cm}$ & $5-10 \mathrm{~cm}$ & $10-20 \mathrm{~cm}$ & Média \\
\hline & \multicolumn{4}{|c|}{ Teor de C $\left(\mathrm{g} \cdot \mathrm{kg}^{-1}\right)$} \\
\hline Floresta Secundária & $5,97 \mathrm{Aa}$ & $4,44 \mathrm{Bb}$ & $3,33 \mathrm{Bc}$ & $4,58 \mathrm{c}$ \\
\hline Pastagem & $6,54 \mathrm{Aa}$ & $5,78 \mathrm{Aa}$ & $4,60 \mathrm{Ab}$ & $5,64 \mathrm{a}$ \\
\hline Teca 19 - entrelinha & $5,37 \mathrm{Aa}$ & $3,95 \mathrm{Bb}$ & $3,52 \mathrm{Bb}$ & $4,28 \mathrm{c}$ \\
\hline Teca 19 - projeção copa & $5,62 \mathrm{Aa}$ & $4,23 \mathrm{Bb}$ & $3,92 \mathrm{Bb}$ & $4,59 \mathrm{c}$ \\
\hline Teca 16 - entrelinha & $5,57 \mathrm{Aa}$ & $4,95 \mathrm{Aa}$ & 4,43 Aa & $4,98 \mathrm{~b}$ \\
\hline Teca 16 - projeção copa & $6,22 \mathrm{Aa}$ & $4,79 \mathrm{Ab}$ & $4,04 \mathrm{Bb}$ & $5,01 \mathrm{~b}$ \\
\hline Teca 13 - entrelinha & $4,68 \mathrm{Aa}$ & $3,97 \mathrm{Ba}$ & $3,67 \mathrm{Ba}$ & $4,11 \mathrm{c}$ \\
\hline Teca 13 - projeção copa & $4,70 \mathrm{Aa}$ & $3,60 \mathrm{Bb}$ & $3,30 \mathrm{Bb}$ & $3,87 \mathrm{c}$ \\
\hline Teca 8 - entrelinha & $5,53 \mathrm{Aa}$ & $5,41 \mathrm{Aa}$ & 4,48 Aa & $5,14 \mathrm{~b}$ \\
\hline Teca 8 - projeção copa & $5,81 \mathrm{Aa}$ & $6,05 \mathrm{Aa}$ & $5,04 \mathrm{Aa}$ & $5,63 \mathrm{a}$ \\
\hline Média & $5,60 \mathrm{a}$ & $4,72 \mathrm{~b}$ & $4,03 \mathrm{c}$ & $\mathrm{CV} 1(\%)=13,57$ \\
\hline CV2(\%) & & & & \\
\hline
\end{tabular}

$\mathrm{CV} 1 \%$ = coeficiente de variação para tratamentos; $\mathrm{CV} 2 \%$ = coeficiente de variação para profundidades. Médias seguidas de mesma letra, maiúscula na coluna e minúscula na linha, não diferem entre si pelo teste Scott-Knott $5 \%$.

$\mathrm{CV} 1 \%$ = coefficient of variation for treatments; CV2\% = coefficient of variation for depths. Means followed by the same letter, uppercase in the column and lowercase in the line, do not differ by the Scott-Knott test at $5 \%$.

Portanto, as áreas de pastagem e de teca 8 anos na projeção da copa foram as que apresentaram os maiores teores de COT, superiores ao sistema natural, além de maior equilíbrio nos teores de $\mathrm{C}$ entre as camadas do solo nos sistemas teca 16, 13 e 8 anos. Isso indica que esses sistemas possuem mecanismos de manutenção de $\mathrm{C}$, possibilitando que este não seja perdido para a atmosfera, contribuindo para o efeito estufa, semelhante ao que ocorre no sistema natural ao manter a ciclagem de nutrientes sem intervenção ao solo.

No caso da pastagem, esse resultado pode estar relacionado ao tipo de gramínea, pois embora não se tenha diversidade de material vegetal a ser decomposto, as gramíneas são capazes de manter ou de aumentar os teores de $\mathrm{C}$, isto porque possuem sistema radicular de crescimento rápido, que agrega as partículas do solo, mantendo a porosidade e, consequentemente, a qualidade física do mesmo e, ainda, à capacidade em sequestrar maior quantidade de $\mathrm{C}$ atmosférico.

Silva et al. (2012) também observaram um dos maiores valores de COT na área de pastagem, atribuindo-o à eficiência de ciclagem de nutrientes da área.
Segundo Barreto et al. (2006), as gramíneas são plantas $\mathrm{C} 4 \mathrm{e}$, devido à sua fisiologia, podem contribuir com maior aporte de $\mathrm{C}$ no solo. Assim, a presença de gramíneas no plantio direto favorece a formação de agregados mais estáveis, aumentando os teores de $\mathrm{C}$ no solo e a taxa de sequestro de $\mathrm{CO}_{2}$ (Salton et al., 2008).

No sistema teca, pode estar relacionado ao tipo de material depositado na superfície do solo e à sua facilidade ou não de decomposição, potencialmente mais lignificado. Além disso, o tempo de deposição e a quantidade de serapilheira formada também favorecem, já que nesse sistema não houve revolvimento do solo e/ou retirada da mesma. Resultados reforçados por Loss et al. (2010), que observaram os maiores valores de COT em área onde se manteve a cobertura morta proveniente de material roçado da vegetação de gramíneas (Paspalum notatum), pois caso os sistemas não sofram revolvimento, haverá uma melhora nas propriedades físicas, químicas e biológicas do solo, o que reduz as perdas por erosão hídrica e oxidação microbiana (Bayer et al., 2004). 


\subsection{Nitrogênio Total}

Os valores obtidos de NT, apresentados na Tabela 3, permitem descrever o decréscimo nos teores do elemento com o aumento da profundidade nos sistemas floresta e teca 13 anos entrelinha, como observado por Freixo et al. (2002), Pulrolnik et al. (2009) e Pimentel (2012), isto pode estar relacionado ao decréscimo de COT em profundidade, provavelmente em decorrência da redução de MOS.
Segundo Freixo et al. (2002), a maior parte dos resíduos orgânicos permanece na superfície do solo, aumentando, assim, com o acúmulo de MO em superfície. Nos demais tratamentos, os teores foram semelhantes aos da Floresta Secundária. Esses resultados podem indicar, no caso do sistema pastagem, que esta foi bem manejada. Rangel e Silva (2007) também observaram pouca diferença nos teores de NT entre os tratamentos testados.

Tabela 3. Teores de nitrogênio total ( $\left.\mathrm{g} \cdot \mathrm{kg}^{-1}\right)$ em função dos sistemas de cultivo e das profundidades de amostragem.

Table 3. Total nitrogen $\left(\mathrm{g} \cdot \mathrm{kg}^{-1}\right)$ in function of cultivation systems and sampling depths.

\begin{tabular}{|c|c|c|c|c|}
\hline \multirow{2}{*}{ Tratamento } & $0-5 \mathrm{~cm}$ & $5-10 \mathrm{~cm}$ & $10-20 \mathrm{~cm}$ & Média \\
\hline & \multicolumn{4}{|c|}{ Teor de N (g.kg-1 $)$} \\
\hline Floresta Secundária & $1,33 \mathrm{Aa}$ & $1,00 \mathrm{Aa}$ & $0,66 \mathrm{Ab}$ & $1,00 \mathrm{a}$ \\
\hline Pastagem & $1,00 \mathrm{Aa}$ & $1,00 \mathrm{Aa}$ & $1,00 \mathrm{Aa}$ & $1,00 \mathrm{a}$ \\
\hline Teca 19 - entrelinha & $1,00 \mathrm{Aa}$ & $1,00 \mathrm{Aa}$ & $1,00 \mathrm{Aa}$ & $1,00 \mathrm{a}$ \\
\hline Teca 19 - projeção copa & $1,00 \mathrm{Aa}$ & $1,00 \mathrm{Aa}$ & $1,00 \mathrm{Aa}$ & $1,00 \mathrm{a}$ \\
\hline Teca 16 - entrelinha & $1,00 \mathrm{Aa}$ & $1,00 \mathrm{Aa}$ & $1,00 \mathrm{Aa}$ & $1,00 \mathrm{a}$ \\
\hline Teca 16 - projeção copa & $1,00 \mathrm{Aa}$ & $1,00 \mathrm{Aa}$ & $0,66 \mathrm{Aa}$ & $0,89 \mathrm{a}$ \\
\hline Teca 13 - entrelinha & $1,00 \mathrm{Aa}$ & $0,66 \mathrm{Ba}$ & $0,00 \mathrm{Bb}$ & $0,55 \mathrm{~b}$ \\
\hline Teca 13 - projeção copa & $0,33 \mathrm{Ba}$ & $0,33 \mathrm{Ba}$ & $0,00 \mathrm{Ba}$ & $0,22 \mathrm{c}$ \\
\hline Teca 8 - entrelinha & $1,00 \mathrm{Aa}$ & $1,00 \mathrm{Aa}$ & $1,00 \mathrm{Aa}$ & $1,00 \mathrm{a}$ \\
\hline Teca 8 - projeção copa & $1,00 \mathrm{Aa}$ & $1,00 \mathrm{Aa}$ & $1,00 \mathrm{Aa}$ & $1,00 \mathrm{a}$ \\
\hline Média & $0,97 \mathrm{a}$ & $0,90 \mathrm{a}$ & 0,73 & $\mathrm{CV} 1(\%)=6,49$ \\
\hline CV2(\%) & \multicolumn{4}{|c|}{5,80} \\
\hline
\end{tabular}

$\mathrm{CV} 1 \%$ = coeficiente de variação para tratamentos; $\mathrm{CV} 2 \%$ = coeficiente de variação para profundidades. Médias seguidas de mesma letra, maiúscula na coluna e minúscula na linha, não diferem entre si pelo teste Scott-Knott 5\%.

$\mathrm{CV} 1 \%$ = coefficient of variation for treatments; CV2\% = coefficient of variation for depths. Means followed by the same letter, uppercase in the column and lowercase in the line, do not differ by the Scott-Knott test at 5\%.

Isso corrobora os resultados observados para os teores de $\mathrm{C}$, demonstrando que os sistemas estudados possuem mecanismos de manutenção ou de aporte e decomposição da MO, o que garante teores de $\mathrm{C}$ e de $\mathrm{N}$ semelhantes aos do sistema natural, apesar da perda de diversidade vegetal.

No entanto, os menores teores de $\mathrm{N}$ foram observados no sistema teca 13 anos projeção da copa, o que pode estar relacionado aos menores teores de COT que, por sua vez, está contido na MOS. Segundo Rangel e Silva (2007), isso se deve ao fato de que mais de $95 \%$ do NT do solo estão associados à $\mathrm{MO}$, e a $\mathrm{MO}$ no sistema teca é de difícil degradação. No presente caso, os teores obtidos foram semelhantes aos obtidos por Matias et al. (2009) e Freixo et al. (2002), e inferiores aos observados por Costa Junior (2008), Pimentel (2012) e Loss et al. (2013).

\subsection{Relação $\mathrm{C} / \mathrm{N}$}

Com os dados de $\mathrm{N}$ e de COT obteve-se a relação C/N (Tabela 4); segundo Santos (2007), esta pode indicar atividade biológica mais ou menos intensa, além do grau de humificação e estabilidade da MO do solo. Nesse caso, os valores de relação $\mathrm{C} / \mathrm{N}$ foram inferiores a $20 \mathrm{em}$ todos os sistemas e profundidades, o que indica que o processo dominante é a mineralização, favorecendo a decomposição e a liberação de $\mathrm{N}$ para o solo (Moreira e Siqueira, 2002), resultados semelhantes aos observados por Santos (2007), Matias et al. (2009) e Pimentel (2012). 
VIEIRA, C.R. et al. Alterações C/N de um Latossolo Vermelho-Amarelo sob diferenes coberturas vegetais

Tabela 4. Relação $\mathrm{C} / \mathrm{N}$ em função dos sistemas de cultivo e das profundidades de amostragem.

Table 4. C/N relation $\left(\mathrm{g} \cdot \mathrm{kg}^{-1}\right)$ in function of cultivation systems and sampling depths.

\begin{tabular}{ccccc}
\hline \multirow{2}{*}{ Tratamento } & $0-5 \mathrm{~cm}$ & $5-10 \mathrm{~cm}$ & $10-20 \mathrm{~cm}$ & Média \\
\cline { 2 - 5 } & \multicolumn{4}{c}{ Relação C/N } \\
\hline Floresta Secundária & $4,01 \mathrm{Cb}$ & $5,57 \mathrm{Ba}$ & $5,13 \mathrm{Ca}$ & $4,90 \mathrm{c}$ \\
Pastagem & $7,82 \mathrm{Aa}$ & $8,06 \mathrm{Aa}$ & $7,31 \mathrm{Aa}$ & $7,73 \mathrm{a}$ \\
Teca 19- entrelinha & $6,49 \mathrm{Ba}$ & $5,95 \mathrm{Ba}$ & $6,40 \mathrm{Ba}$ & $6,28 \mathrm{~b}$ \\
Teca 19- projeção copa & $6,87 \mathrm{Ba}$ & $6,52 \mathrm{Ba}$ & $6,60 \mathrm{Ba}$ & $6,66 \mathrm{~b}$ \\
Teca 16- entrelinha & $6,97 \mathrm{Ba}$ & $7,02 \mathrm{Aa}$ & $6,50 \mathrm{Ba}$ & $6,83 \mathrm{~b}$ \\
Teca 16- projeção copa & $8,26 \mathrm{Aa}$ & $7,97 \mathrm{Aa}$ & $7,23 \mathrm{Aa}$ & $7,82 \mathrm{a}$ \\
Teca 13- entrelinha & $7,86 \mathrm{Aa}$ & $7,82 \mathrm{Aa}$ & $7,54 \mathrm{Aa}$ & $7,74 \mathrm{a}$ \\
Teca 13- projeção copa & $8,86 \mathrm{Aa}$ & $7,77 \mathrm{Aa}$ & $8,00 \mathrm{Aa}$ & $8,21 \mathrm{a}$ \\
Teca 8- entrelinha & $7,61 \mathrm{Aa}$ & $7,45 \mathrm{Aa}$ & $7,56 \mathrm{Aa}$ & $7,54 \mathrm{a}$ \\
Teca 8- projeção copa & $7,66 \mathrm{Aa}$ & $7,70 \mathrm{Aa}$ & $8,35 \mathrm{Aa}$ & $7,90 \mathrm{a}$ \\
\hline Média & $7,24 \mathrm{a}$ & $7,18 \mathrm{a}$ & $7,06 \mathrm{a}$ & $\mathrm{CV} 1(\%)=10,54$ \\
\hline CV2(\%) & & \multicolumn{3}{c}{} \\
\hline
\end{tabular}

$\mathrm{CV} 1 \%$ = coeficiente de variação para tratamentos; CV2\% = coeficiente de variação para profundidades. Médias seguidas de mesma letra, maiúscula na coluna e minúscula na linha, não diferem entre si pelo teste Scott-Knott 5\%.

$\mathrm{CV} 1 \%$ = coefficient of variation for treatments; CV2\% = coefficient of variation for depths. Means followed by the same letter, uppercase in the column and lowercase in the line, do not differ by the Scott-Knott test at 5\%.

Avaliando-se as camadas do solo, o sistema floresta apresentou os menores valores de relação $\mathrm{C} / \mathrm{N}$ entre os sistemas avaliados, tendendo a aumentar na profundidade $5-10 \mathrm{~cm}$, pois a floresta possui maior diversidade de material vegetal, que, por sua vez, possui diferentes graus de decomposição, ou seja, dos mais aos menos lignificados, contribuindo também para a maior diversidade da microbiota do solo. São eles que degradam a MO, liberando os nutrientes. Nesse caso, a degradação pode ser mais rápida, assim como o aporte de material, mantendo o ciclo, devido a menor relação $\mathrm{C} / \mathrm{N}$.

Com relação aos sistemas de cultivo de teca, o cultivo de 19 anos apresentou os menores valores para a relação $\mathrm{C} / \mathrm{N}$, o que pode estar relacionado ao tempo de deposição do material, enquanto os maiores valores foram observados nos sistemas: pastagem, teca 16 projeção de copa, teca 13 entrelinha e projeção de copa, teca 8 entrelinha e projeção de copa, nestes, o processo de decomposição foi mais lento que na Floresta Secundária. A maior relação $\mathrm{C} / \mathrm{N}$ acarreta degradação mais lenta e favorece o acúmulo de COT e $\mathrm{N}$ nos agregados do solo (Loss et al., 2011).

A exemplo disso, Schiavo et al. (2011) verificaram que a pastagem de braquiária foi a cobertura de solo que proporcionou índices de manejo de $\mathrm{C}$ no solo mais próximos aos da vegetação de Cerrado, enquanto Teixeira et al. (2010) observaram maiores valores de relação $\mathrm{C} / \mathrm{N}$ em plantios de Eucalyptus sp.; segundo os autores, a qualidade dos resíduos influenciou a incorporação de $\mathrm{MO}$, sugerindo menor mineralização. Os autores especularam ainda que, nesse caso, houve favorecimento para perda de $\mathrm{N}$ em forma de gases. $\mathrm{O}$ mesmo pode ter ocorrido no presente estudo devido aos valores de $\mathrm{N}$ obtidos.

Além disso, algumas áreas podem apresentar maior relação $\mathrm{C} / \mathrm{N}$ devido à sua maior acumulação de COT e de $\mathrm{N}$ no perfil do solo, em razão da estabilidade das frações húmicas e menor grau de mineralização da MOS (Campos et al., 2013).

Portanto, os sistemas de cultivo de T. grandis apresentaram valores superiores na relação $\mathrm{C} / \mathrm{N}$, o que se explica pela menor diversidade de material a ser decomposto. No entanto, no presente caso, a relação $\mathrm{C} / \mathrm{N}$ esteve entre valores que propiciam a decomposição da $\mathrm{MO}$, liberando os nutrientes essenciais. Assim, os sistemas de cultivo de $T$. grandis e a pastagem apresentaram semelhança ao sistema nativo, representado pela Floresta Secundária; isto se deve, provavelmente, às poucas intervenções nos solos sob estas culturas. 


\section{CONCLUSÕES}

Os sistemas pastagem e teca (T. grandis) assemelharam-se ao sistema Floresta Secundária, em termos de manutenção nos teores de $\mathrm{C}$ e de $\mathrm{N}$, já que os referidos sistemas de cultivo não apresentaram reduções em relação ao sistema referência.

Os valores de relação $\mathrm{C} / \mathrm{N}$ indicaram que o processo dominante é a mineralização. No entanto, o sistema teca 16 anos foi o único que apresentou alteração dos valores de relação $\mathrm{C} / \mathrm{N}$ entre projeção de copa e entrelinha entre todos os sistemas com plantio de $T$. grandis e a Floresta Secundária apresentou um dos menores valores.

\section{REFERÊNCIAS BIBLIOGRÁFICAS}

BARRETO, A.C. et al. Características químicas e físicas de um solo sob floresta, sistema agroflorestal e pastagem no sul da Bahia. Caatinga, v. 19, n. 4, p. 415-425, 2006.

BAYER, C. et al. Armazenamento de carbono em frações lábeis da matéria orgânica de um Latossolo Vermelho sob plantio direto. Pesquisa Agropecuária Brasileira, v. 39, n. 7, p. 677-683, 2004.

et al. A method for estimating coefficients of soil organic matter dynamics based on long-term experiments. Soil Tillage Research, v. 91, p. 217-226, 2006.

BRASIL. Ministério das Minas e Energia Departamento Nacional de Produção Mineral. Projeto RADAMBRASIL. Folhas SD.23. Rio de Janeiro, 1992. 660 p. (Levantamento de Recursos Naturais, 29).

BREMNER, J.M.; MULVANEY, C.S. Total nitrogen. In: PAGE, A.L. (Ed.). Methods of soil analysis. Madison: American Society of Agronomy, 1982. p. 595-624.

CAMPOLIN, W.R. Características físicas e químicas, estoque de matéria orgânica (total e nas frações granulométricas) do solo sob Cerrado e teca. 2006. 49 f. Dissertação (Mestrado em Agricultura Tropical) - Faculdade de Agronomia, Medicina Veterinária e Zootecnia, Universidade Federal de Mato Grosso, Cuiabá.
CAMPOS, L.P. et al. Estoque e frações de carbono orgânico em Latossolo Amarelo submetido a diferentes sistemas de manejo. Pesquisa Agropecuária Brasileira, v. 48, n. 3, p. 304-312, 2013.

CASTRO, G.C. Carbono orgânico nas frações granulométricas e húmicas em solos de diferentes texturas sob floresta da região noroeste mato-grossense. 2008. 45 f. Dissertação (Mestrado em Ciências Florestais e Ambientais) Faculdade de Engenharia Florestal, Universidade Federal de Mato Grosso, Cuiabá.

COSTA JUNIOR, C. Estoque de carbono e nitrogênio e agregação do solo sob diferentes sistemas de manejo agrícola no Cerrado, em Rio Verde (GO). 2008. 139 f. Dissertação (Mestrado em Ciências) - Centro de Energia Nuclear na Agricultura, Universidade de São Paulo, Piracicaba.

DENARDIN, R.B.N. et al. Estoque de carbono no solo sob diferentes formações florestais, Chapecó SC. Ciência Florestal, v. 24, n. 1, p. 59-69, 2014.

EMPRESA BRASILEIRA DE PESQUISA AGROPECUÁRIA - EMBRAPA. Centro Nacional Pesquisa de Solos. Sistema brasileiro de classificação de solos. Brasília, DF, 1999. 412 p.

FERREIRA, D.F. Sisvar: versão 4.6. Lavras: Universidade Federal de Lavras, 2003.

FRANZLUEBBERS, A.J.; SCHOMBERG, H.H.; ENDALE, D.M. Surface-soil responses to paraplowing of long-term no-tillage cropland in the Southern Piedmont USA. Soil and Tillage Research, v. 96, p. 303-315, 2007.

FREIXO, A.A. et al. Estoques de carbono e nitrogênio e distribuição de frações orgânicas de Latossolo do Cerrado sob diferentes sistemas de cultivo. Revista Brasileira de Ciência do Solo, v. 26, n. 2, p. 425-434, 2002.

LOSS, A. et al. Carbono, matéria orgânica leve e frações oxidáveis do carbono orgânico sob diferentes sistemas de produção orgânica. Comunicata Scientiae, v. 1, n. 1, p. 57-64, 2010.

et al. Agregação, carbono e nitrogênio em agregados do solo sob plantio com integração lavoura-pecuária. Pesquisa Agropecuária Brasileira, v. 46, n. 10, p. 1269-1276, 2011. 
VIEIRA, C.R. et al. Alterações $\mathrm{C} / \mathrm{N}$ de um Latossolo Vermelho-Amarelo sob diferenes coberturas vegetais

LOSS, A. et al. Carbono mineralizável, carbono orgânico e nitrogênio em macroagregados de Latossolo sob diferentes sistemas de uso do solo no Cerrado Goiano. Semina: Ciências Agrárias, v. 34, n. 5, p. 2153-2168, 2013.

MACHADO, P.O. Carbono do solo e a mitigação da mudança climática global. Química Nova, v. 28, n. 2, p. 239-334, 2005.

MATIAS, M.C.B.S. et al. Biomassa microbiana e estoques de $\mathrm{C}$ e $\mathrm{N}$ do solo em diferentes sistemas de manejo, no Cerrado do Estado do Piauí. Acta Scientiarum. Agronomy, v. 31, n. 3, p. 517-521, 2009.

MATIAS, S.S.R. et al. Influência de diferentes sistemas de cultivo nos atributos físicos e no carbono orgânico do solo. Revisa Brasileira de Ciência Agrária, v. 7, n. 3, p. 414-420, 2012.

MOREIRA, A.; MALAVOLTA, E. Dinâmica da matéria orgânica e da biomassa microbiana em solo submetido a diferentes sistemas de manejo na Amazônia Ocidental. Pesquisa Agropecuária Brasileira, v. 39, n. 11, p. 1103-1110, 2004.

MOREIRA, F.M.; SIQUEIRA, J.O. Microbiologia e bioquímica do solo. Lavras: Universidade Federal de Lavras, 2002. 625 p.

PIMENTEL, R.M. Propriedades físicas, carbono e nitrogênio do solo em sistemas agropecuários. 2012. 99 f. Dissertação (Mestrado em Zootecnia) Departamento de Zootecnia, Universidade Federal de Lavras, Lavras.

PULROLNIK, K. et al. Estoques de carbono e nitrogênio em frações lábeis e estáveis da matéria orgânica de solos sob eucalipto, pastagem e Cerrado no Vale do Jequitinhonha - MG. Revista Brasileira de Ciência do Solo, v. 33, n. 5, p. 1125-1136, 2009.

RANGEL, O.J.P.; SILVA, C.A. Estoques de carbono e nitrogênio e frações orgânicas de Latossolo submetido a diferentes sistemas de uso e manejo. Revista Brasileira de Ciência do Solo, v. 31, n. 6, p. 1609-1623, 2007.

SALTON, J.C. et al. Agregação e estabilidade de agregados do solo em sistemas agropecuários em Mato Grosso do Sul. Revista Brasileira de Ciência do Solo, v. 32, n. 1, p. 11-21, 2008.
SANTOS, C.H. Alterações no estoque de carbono, na dinâmica da matéria orgânica e dos atributos químicos e físicos induzidas por diferentes usos dos solos de Colorado do Oeste-RO. 2008. 76 f. Dissertação (Mestrado em Agricultura Tropical) - Faculdade de Agronomia, Medicina Veterinária e Zootecnia, Universidade Federal de Mato Grosso, Cuiabá.

SANTOS, E. Carbono, nitrogênio e relação C/N em Gleissolo e Cambissolo sob diferentes tipologias vegetais na área de ocorrência da Floresta Ombrófila Densa, Antonina - PR. 2007. 104 f. Dissertação (Mestrado em Ciência do Solo) Setor de Ciências Agrárias, Universidade Federal do Paraná, Curitiba.

SCHIAVO, J.A. et al. Índice de manejo de carbono e atributos químicos de Latossolo Vermelho sob diferentes sistemas de manejo. Pesquisa Agropecuária Brasileira, v. 46, n. 10, p. 1332-1338, 2011.

SILVA, C.F. et al. Carbono orgânico total, biomassa microbiana e atividade enzimática do solo de áreas agrícolas, florestais e pastagem no médio Vale do Paraíba do Sul (RJ). Revista Brasileira de Ciência do Solo, v. 36, n. 6, p. 1680-1689, 2012.

SOUZA, E.D. et al. Frações do carbono orgânico, biomassa e atividade microbiana em um Latossolo Vermelho sob cerrado submetido a diferentes sistemas de manejos e usos do solo. Acta Scientiarum. Agronomy, v. 28, n. 3, p. 323-329, 2006.

TEIXEIRA, J.M. et al. Estoque de nitrogênio total e relação $\mathrm{C} / \mathrm{N}$ influenciados pelo sistema de manejo e uso do solo de Cerrado. In: CONGRESSO NACIONAL DE MILHO E SORGO, 28., 2010, Goiânia. Anais... Goiânia: Associação Brasileira de Milho e Sorgo, 2010. CD-Rom.

YEOMANS, A.; BREMNER, J.M. A rapid and precise method for routine determination of organic carbon in soil. Communication in Soil Science and Plant Analysis, v. 19, p. 1467-1476, 1988. 